\title{
Routine use of clinical exome-based next-generation sequencing for evaluation of patients with thrombotic microangiopathies
}

Joseph P Gaut ${ }^{1,2}$, Sanjay Jain ${ }^{1,2}$, John D Pfeifer ${ }^{1}$, Katinka A Vigh-Conrad ${ }^{1}$, Meagan Corliss ${ }^{1}$, Mukesh K Sharma ${ }^{1}$, Jonathan W Heusel ${ }^{1,3}$ and Catherine E Cottrell ${ }^{1,3,4}$

${ }^{1}$ Department of Pathology and Immunology, Division of Anatomic and Molecular Pathology, Washington University School of Medicine, St Louis, MO, USA; ${ }^{2}$ Department of Medicine, Washington University School of Medicine, Renal Division, St Louis, MO, USA; ${ }^{3}$ Department of Genetics, Washington University School of Medicine, St Louis, MO, USA and ${ }^{4}$ Institute for Genomic Medicine, Nationwide Children's Hospital, St Louis, $M O, U S A$

\begin{abstract}
Next-generation sequencing is increasingly used for clinical evaluation of patients presenting with thrombotic microangiopathies because it allows for simultaneous interrogation of multiple complement and coagulation pathway genes known to be associated with disease. However, the diagnostic yield is undefined in routine clinical practice. Historic studies relied on case-control cohorts, did not apply current guidelines for variant pathogenicity assessment, and used targeted gene enrichment combined with next-generation sequencing. A clinically enhanced exome, targeting $\sim 54 \mathrm{Mb}$, was sequenced for 73 patients. Variant analysis and interpretation were performed on genes with biological relevance in thrombotic microangiopathy $(C 3, C D 46, C F B, C F H, C F I$, $D G K E$, and THBD). CFHR3-CFHR1 deletion status was also assessed using multiplex ligation-dependent probe amplification. Variants were classified using American College of Medical Genetics and Genomics guidelines. We identified 5 unique novel and 14 unique rare variants in $25 \%$ (18/73) of patients, including a total of 5 pathogenic, 4 likely pathogenic, and 15 variants of uncertain clinical significance. Nine patients had homozygous deletions in CFHR3-CFHR1. The diagnostic yield, defined as the presence of a pathogenic variant, likely pathogenic variant or homozygous deletion of CFHR3-CFHR1, was $25 \%$ for all patients tested. Variants of uncertain clinical significance were identified in $21 \%(15 / 73)$ of patients.These results illustrate the expected diagnositic yield in the setting of thrombotic microangiopathies through the application of standardized variant interpretation, and highlight the utility of such an approach. Sequencing a clinically enhanced exome to enable targeted, disease-specific variant analysis is a viable approach. The moderate rate of variants of uncertain clinical significance highlights the paucity of data surrounding the variants in our cohort and illustrates the need for expanded variant curation resources to aid in thrombotic microangiopathy-related disease variant classification. Modern Pathology (2017) 30, 1739-1747; doi:10.1038/modpathol.2017.90; published online 28 July 2017
\end{abstract}

Thrombotic microangiopathies, defined by microangiopathic hemolytic anemia, microvascular thrombosis, and organ damage, are a diverse group of disorders with varied etiologies. ${ }^{1,2}$ Atypical hemolytic uremic syndrome is an event-triggered thrombotic microangiopathy due to overactivation of the

Correspondence: Assistant Professor JP Gaut, MD, PhD, Department of Pathology and Immunology, Division of Anatomic and Molecular Pathology, Washington University School of Medicine, Campus Box 8118, 660 South Euclid Avenue, St Louis, MO 631101093, USA.

E-mail: jpgaut@ wustl.edu

Received 31 March 2017; revised 24 May 2017; accepted 25 May 2017; published online 28 July 2017 alternative complement pathway predominantly involving the kidneys. ${ }^{1,3}$ Hereditary and acquired genetic variants in the alternative complement and coagulation pathways have been identified in association with disease. ${ }^{1,4-9}$ Owing to the ability to evaluate multiple genes simultaneously for known and novel mutations, massively parallel or 'nextgeneration' sequencing is poised to have a critical role in identifying patients with thrombotic microangiopathy-related genetic variants that have implications for diagnosis, therapy, and prognosis..$^{3,7,10-14}$ Clinical practice guidelines recommend genetic testing for all patients presenting with atypical hemolytic uremic syndrome to guide prognosis and treatment. ${ }^{15-17}$ 
Historically, when a genetic etiology was considered, single-gene analysis was used to identify variants associated with clinical phenotypes. As the number of causative genes grows, this iterative process becomes costly and time-consuming. The advent of rapid, economical, and efficient nextgeneration sequencing technologies has revolutionized medical practice. Since completion of the initial draft of the human genome in 2000, the cost and time required to sequence the human genome have decreased markedly. ${ }^{18}$ Additional improvements in accuracy and automation allowed nextgeneration sequencing to become a widespread clinical diagnostic tool for pathogenic variant detection. Several reports described the utility of targeted gene enrichment combined with next-generation sequencing in renal disease evaluation. ${ }^{14,19-23}$ Using this approach, investigators reported a positive genetic diagnosis in $43-61 \%$ of patients sequenced for atypical hemolytic uremic syndrome. ${ }^{3,4,7,24}$ However, none of these studies used application of current variant interpretation standards as defined by the American College of Medical Genetics and Genomics/American Molecular Pathology Association (ACMG/AMP). ${ }^{25}$

The development of the clinical exome holds significant promise for genetic testing in patients with constitutional disease. The clinical research exome has become a dominant exome-capture reagent for clinical next-generation sequencing in the assessment of constitutional disease because it

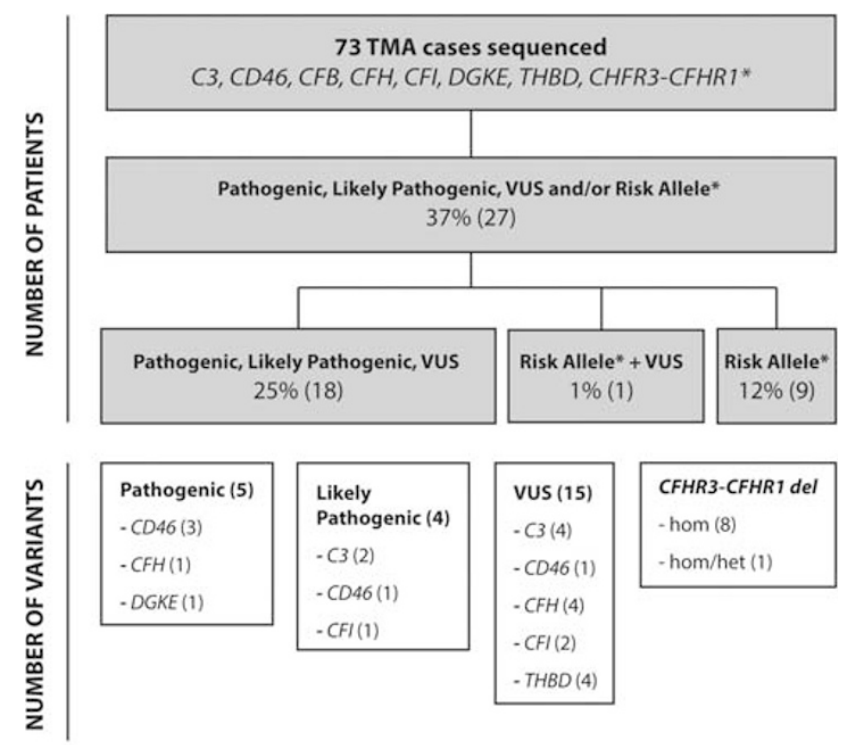

VUS: variants of unknown significance

*homozygous CFHR3-CFHR1 deletion (analyzed by MLPA)

Figure 1 Flow chart depicting the thrombotic microangiopathy genetic testing results by the number of patients with variants, variant interpretation, and variant distribution among complement and coagulation pathway genes. TMA, thrombotic microangiopathy; VUS, variant of uncertain clinical significance. provides enrichment for more than 4600 genes known to be associated with complex and Mendelian disease as curated from databases including Online Mendelian Inheritance in Man, ClinVar, and the Human Gene Mutation Database. This represents a cost-effective technique for the generation of hundreds of disease-specific gene panels that is readily adaptable as clinical knowledge advances. With approved human research protocols, variants across the exome may be evaluated for discovery and other clinical research investigations. To our knowledge, no prior studies have used clinical exome sequencing followed by targeted, disease-specific bioinformatic analysis and reporting in the evaluation of patients with thrombotic microangiopathies.

This study is the first to report the diagnostic yield of next-generation sequencing testing using a clinical exome-based strategy and rigorous application of current clinical variant interpretation standards for patients with thrombotic microangiopathies in routine clinical practice. Our results demonstrate that this approach identifies a genetic cause, defined as identification of pathogenic variants or likely pathogenic variants in $12 \%$ of all patients referred for thrombotic microangiopathy genetic testing. The diagnostic yield increases to $25 \%$ by including detection of homozygous deletion of CFHR3CFHR1. This is significant as most cases in this cohort referred for clinical sequencing represent singletons with little clinical data provided.

\section{Materials and methods}

\section{Laboratory Workflow}

This study was granted exempt status from the Washington University School of Medicine institutional review board. All tests were requested by licensed physicians (Figure 1). The data presented represent consecutive samples submitted to Genomics and Pathology Services at Washington University in Saint Louis (GPS@WUSTL) between 1 February 2015 and 29 February 2016. The laboratory is College of American Pathologists (CAP)-accredited and Clinical Laboratory Improvement Amendments (CLIA)-certified. Test methodology was twofold, encompassing both next-generation sequencing for genetic variant detection, and multiplex ligationdependent probe amplification for detection of deletion of CFHR1-CFHR3. The submitted specimen for testing represented $2-5 \mathrm{ml}$ of peripheral blood obtained in a lavender-top EDTA tube with genomic DNA extracted manually using the QIAamp DNA Blood Mini Kit (Qiagen, Valencia, CA). Deletion analysis was performed on extracted DNA as a sendout test to Cincinnati Children's Hospital. Briefly, oligonucleotide probes hybridize adjacent regions of the CFHR1 and CFHR3 genes. Only probes that are adjacently hybridized are able to be ligated, 
amplified, and quantified using capillary electrophoresis. In the presence of CFHR1-CFHR3 deletions, probe amplification will be diminished or absent. $^{26,27}$ Library preparation for the nextgeneration sequencing assay began by fragmenting DNA to 200 bp by ultrasonication, followed by end repair, A-tailing, and ligation to sequencing adapters. Target capture was performed using the SureSelectXT Clinical Research Exome (Agilent Technologies, Santa Clara, CA) encompassing $54 \mathrm{Mb}$ of target space. Libraries were sequenced using an Illumina HiSeq2500 (Illumina, San Diego, CA) with paired $2 \times 101$-bp reads. Analytic sensitivity, specificity, and reproducibility were established per the CAP next-generation sequencing-testing checklist. ${ }^{28}$

\section{Variant Annotation and Reporting}

Variant classifications were based on standards and guidelines presented as a joint consensus recommendation published by the American College of Medical Genetics and Genomics and the Association of Molecular Pathology. ${ }^{25}$ Variant calls were reported using Human Genome Variation Society nomenclature (http://www.hgvs.org/mutnomen), and variant attributes were examined using sequence variation databases including the Exome Aggregation Consortium (ExAC) (exac.broadinstitute.org, encompassing data from the 1000 Genomes project, and NHLBI-GO Exome Sequencing Project, among others, along with annotated data from the SingleNucleotide Polymorphism Database (v135) (http:// www.ncbi.nlm.nih.gov/projects/SNP/)), the FH atypical hemolytic uremic syndrome mutation database (http://www.fh-hus.org/), ClinVar (http://www.ncbi. nlm.nih.gov/clinvar/), the HGMD public resource (http://www.hgmd.cf.ac.uk/ac/index.php), and an internally curated clinical-grade database of variants and interpretations housed in the Clinical Genomicist Workspace (PierianDx, St Louis, MO). ${ }^{29,30}$ Variants were classified on the basis of the sum of evidence surrounding the genomic alteration: level 1-pathogenic, level 2-likely pathogenic, level 3variant of uncertain significance, level 4 likely benign, and level 5-benign. ${ }^{25}$ All results were reviewed by a pathologist with subspecialty boards in Molecular Genetics from the American Board of Pathology, or a clinical laboratory geneticist certified in Clinical Molecular Genetics and Clinical Cytogenetics from the American Board of Medical Genetics and Genomics before release to the patient's medical record.

\section{Statistics}

Statistical analyses were performed using the GraphPad Software. Categorical variables were examined using the Fisher's exact test, two-tailed. Continuous variables were evaluated using a Mann-Whitney test.

\section{Results}

\section{Assay Design and Validation}

The thrombotic microangiopathy genetic test is performed using the Agilent SureSelectXT Clinical Research exome with the reported gene set encompassing CFH, CFI, CFB, C3, CD46, DGKE, and THBD. In total, the capture reagent targets $\sim 54 \mathrm{Mb}$ of exonic and selected intronic regions for sequencing. Bioinformatic filtering is used to restrict the analyzed and reported gene set. Validation was performed using 28 genomic DNA samples including 3 HapMap cell lines provided by the National Institute of General Medical Sciences-Coriell cell repositories, 5 blinded peripheral blood samples from individuals without a history of renal disease, 17 blinded patient samples harboring at least one known pathogenic genetic variant, a single unblinded patient sample, and 2 production patient samples. All analyses were based on human reference sequence UCSC build hg19; NCBI build 37.2. Analytic sensitivity, specificity, and positive predictive value to detect single base pair variation in coding regions throughout the entire Agilent SureSelectXT Clinical Research exome were $\sim 96.6 \%, 100.0 \%$, and $99.3 \%$, respectively, as determined by comparison of genetic sequence called by this assay using HapMap DNA sample NA12878 to the high-confidence genotypes reported at those positions by Complete Genomics (http://www.completegenomics.com). Analysis of the subset of thrombotic microangiopathy disease-associated genes showed sensitivity, specificity, and positive predictive value, all approaching $100 \%$. Singlenucleotide variants and small insertion/deletion events were called using SAMtools mpileup. ${ }^{31,32}$

\section{Patient Characteristics}

Seventy-three patients underwent exome-based thrombotic microangiopathy genetic testing. The demographic characteristics are summarized in Table 1 . The average age at the time of testing was $36.7 \pm 16.5$ years. Forty-six females and 27 males were tested. Patients were classified based on the referring clinician's clinical diagnosis, which included 61 atypical hemolytic uremic syndrome, 4 thrombotic thrombocytopenic purpura, and 8 thrombotic microangiopathy.

\section{Variant Interpretation}

Before variant calling and interpretation, all samples underwent detailed quality control analysis. ${ }^{28} \mathrm{~A}$ quality control metrics report is generated detailing the total number of reads, percent mapped to the genome, on-target, on-target unique, mean mapping quality, and depth of coverage. Genomic positions failing to meet a coverage depth of at least 10x, or those failing to meet a variant quality call of 30 , were 
Table 1 Patient demographics

\begin{tabular}{|c|c|c|c|c|}
\hline Diagnosis & $\begin{array}{l}\text { Number } \\
\text { of } \\
\text { patients }\end{array}$ & Age (years) & Gender & Race \\
\hline aHUS/HUS & 61 & $35.5 \pm 16.2$ & $22 \mathrm{M}: 39 \mathrm{~F}$ & $\begin{array}{l}37 \text { Caucasian } \\
5 \text { Hispanic } \\
6 \text { African } \\
\text { American } \\
4 \text { Asian } \\
1 \\
\text { Mediterranean } \\
3 \text { Mixed } \\
5 \text { Unknown }\end{array}$ \\
\hline TTP & 4 & $47.8 \pm 3.3$ & $2 \mathrm{M}: 2 \mathrm{~F}$ & $\begin{array}{l}3 \text { Caucasian } \\
1 \text { African } \\
\text { American }\end{array}$ \\
\hline TMA & 8 & $40.9 \pm 21$ & $3 \mathrm{M}: 5 \mathrm{~F}$ & $\begin{array}{l}5 \text { Caucasian } \\
1 \text { Hispanic } \\
1 \text { African } \\
\text { American } \\
1 \text { Asian }\end{array}$ \\
\hline All patients & 73 & $36.7 \pm 16.5$ & $27 \mathrm{M}: 46 \mathrm{~F}$ & $\begin{array}{l}45 \text { Caucasian } \\
6 \text { Hispanic } \\
8 \text { African } \\
\text { American } \\
5 \text { Asian } \\
1 \\
\text { Mediterranean } \\
3 \text { Mixed } \\
5 \text { Unknown }\end{array}$ \\
\hline
\end{tabular}

Abbreviations: aHUS, atypical hemolytic uremic syndrome; HUS, hemolytic uremic syndrome; TMA, thrombotic microangiopathy; TTP, thrombotic thrombocytopenic purpura.

filtered out of analysis. All variants were initially filtered for a population minor allele frequency $<5 \%$; this cutoff was chosen as this represents stand-alone evidence for a benign classification according to ACMG/AMP guidelines. ${ }^{25}$ All variants with a minor allele frequency $<5 \%$ were analyzed and scored as pathogenic, likely pathogenic, uncertain clinical significance, likely benign, or benign based on the scheme outlined by the ACMG/AMP. ${ }^{25}$ To facilitate classification, a variant assessment worksheet with the ACMG/AMP criteria is used for each variant and archived as part of the permanent testing record.

\section{Genetic Variants}

The genetic variants are summarized in Table 2. Of the 73 patients sequenced, $27 \%$ (20/73) harbored 24 variants classified as variants of uncertain clinical significance, pathogenic, or likely pathogenic, and $12 \%(9 / 73)$ had homozygous deletions in CFHR3CFHR1. Among the 24 single-nucleotide variants are 5 pathogenic variants, 4 likely pathogenic variants, and 15 variants of uncertain clinical significance (Figure 1). Six variants (5 of which are unique) are novel, having not been reported in the literature or deposited in existing population or diseaseassociated variant databases (ExAC, NHLBI, FHaHUS, dbSNP). Details of the pathogenic/likely pathogenic variants are summarized in Table 3 and variants of uncertain clinical significance are summarized in Table 4. Six patients carried multiple genomic alterations: greater than one singlenucleotide variant and/or harboring a homozygous CFHR3-CFHR1 deletion (Figure 2). The patients identified with pathogenic or likely pathogenic variants tended to be younger at the time of testing, $29.2 \pm 14.5$ years compared with $37.8 \pm 16.6$ years. However, this difference was not statistically significant.

Uniquely occuring pathogenic and likely pathogenic variants $(n=8)$ classified by the ACMG/AMP criteria were compared with unique variants of uncertain clinical significance $(n=12)$ classified using the same criteria. Pathogenic and likely pathogenic variants tended to have lower minor allele frequencies across all populations based on the ExAC database, $1.11 \mathrm{e}-05 \times 2.18 \mathrm{e}-05$ compared with variants of uncertain clinical significance, $7.35 \mathrm{e}-$ $04 \times 1.32 \mathrm{e}-03(P=0.11) .{ }^{30}$ The genomic evolutionary rate profiling $(\mathrm{GERP}++)$ scores quantifying evolutionary constraint differed significantly between the variant classifications, $4.17 \pm 1.86$ for pathogenic/ likely pathogenic versus $-0.16 \pm 3.73$ for variants of uncertain clinical significance $(P=0.004) .{ }^{33}$ In silico prediction tools were compared for the missense variants classified as pathogenic/likely pathogenic $(n=4)$ and variants of uncertain clinical significance $(n=12)$. SIFT (release 63), PolyPhen-2 (version 2.2.2, r394), and the protein variation effect analyzer (PROVEAN, version 1.1.5) algorithms showed statistically significant differences between the pathogenic/ likely pathogenic variants compared with variants of uncertain clinical significance, $-1.80 \pm 2.10$ $\left(P=0.001, P=0.03\right.$, and $P=0.004$, respectively.$^{34}$

$C F H$ and $C D 46$ were most commonly identified as harboring single-nucleotide variants, each occurring in $7 \%(5 / 73)$ of patients (Figures 1 and 2). The five patients with $\mathrm{CFH}$ variants had four distinct singlenucleotide variants, three classified as variants of uncertain clinical significance and one as pathogenic (Tables 3 and 4). Two were reported previously. ${ }^{24,35-39}$ One patient with the p.Q950H variant of uncertain clinical significance also showed homozygous deletion of CFHR3-CFHR1. Two related patients carrying the p.G918E $C F H$ variant also harbored the c.287-2A $>$ G splice-site pathogenic variant in CD46. CD46 contained four distinct variants (Figures 1 and 2). Of the four variants, two were classified as pathogenic, one likely pathogenic, and one of uncertain clinical significance (Tables 3 and 4).

Three distinct variants were found in CFI, one likely pathogenic and two of uncertain clinical significance (Figure 2 and Table 3). The two variants of uncertain clinical significance have been reported 
Table 2 Genetic variants meeting criteria for pathogenic, likely pathogenic, or VUS identified in patients referred for TMA genetic testing

\begin{tabular}{|c|c|c|c|c|c|c|}
\hline Patient & Gene & Interpretation & Variant (coding) & Variant (genomic) & Tуре & Clinical diagnosis \\
\hline 65 & C3 & LP & p.R1042L & Chr19:g.6694471C >A & Missense & aHUS \\
\hline 7 & C3 & LP & p.R161W & Chr19:g.6718128G >A & Missense & aHUS \\
\hline 3 & C3 & VUS & p.T647Ma & Chr19:g.6707846G > A & Missense & aHUS \\
\hline 40 & C3 & VUS & p.Q185H & Chr19:g.6714407C > G & Missense & aHUS \\
\hline 80 & C3 & VUS & p.T1383N & Chr19:g.6684423G > T & Missense & aHUS \\
\hline 78 & C3 & VUS & p.E1636G & Chr19:g.6677978T $>$ C & Missense & aHUS \\
\hline 12 & CD46 & $\mathrm{P}$ & c. $287-2 A>G$ & Chr1:g.207930883A > G & Splice site & HUS \\
\hline 35 & $C D 46$ & $\mathrm{P}$ & c. $287-2 \mathrm{~A}>\mathrm{G}$ & Chr1:g.207930883A $>$ G & Splice site & Postpartum TMA \\
\hline 25 & $C D 46$ & $\mathrm{P}$ & c. $1127+2 \mathrm{~T}>\mathrm{G}^{\mathrm{a}}$ & Chr1:g.207959029T > G & Splice site & TTP \\
\hline 5 & CD46 & LP & p.C35Y & Chr1:g.207930365G $>A$ & Missense & HUS \\
\hline 38 & $C D 46$ & VUS & p.P324L & Chr1:g.207943690C $>\mathrm{T}$ & Missense & aHUS s/p renal transplant \\
\hline 21 & $\mathrm{CFH}$ & $\mathrm{P}$ & c. $619+1 G>A^{a}$ & Chr1:g.196646798G > A & Splice site & aHUS \\
\hline 84 & $\mathrm{CFH}$ & VUS & p.I372V $\mathrm{V}^{\mathrm{a}}$ & Chr1:g.196658699A $>$ G & Missense & TMA, loss of two kidney transplants \\
\hline 31 & $\mathrm{CFH}$ & VUS & p.Q950H & Chr1:g.196709816G $>\mathrm{T}$ & Missense & aHUS \\
\hline 12 & CFH & VUS & p.G918E & Chr1:g.196706761G >A & Missense & HUS \\
\hline 35 & $\mathrm{CFH}$ & VUS & p.G918E & Chr1:g.196706761G $>$ A & Missense & Postpartum TMA \\
\hline 69 & CFI & LP & p.I370Na & Chr4:g.110670413A > T & Missense & aHUS \\
\hline 30 & CFI & VUS & p.I306V & Chr4:g.110673648T $>$ C & Missense & HUS \\
\hline 45 & CFI & VUS & p.D403N & Chr4:g.110667600C $>\mathrm{T}$ & Missense & aHUS \\
\hline 79 & $D G K E$ & $\mathrm{P}$ & p.W322* & Chr17:g.54926134G >A & Nonsense & aHUS \\
\hline 27 & $T H B D$ & VUS & p.P495S & Chr20:g.23028659G >A & Missense & HUS \\
\hline 79 & $T H B D$ & VUS & p.A43T & Chr20:g.23030015C > T & Missense & aHUS \\
\hline 42 & $T H B D$ & VUS & p.A43T & Chr20:g.23030015C $>\mathrm{T}$ & Missense & aHUS \\
\hline 65 & $T H B D$ & VUS & p.A43T & Chr20:g.23030015C > T & Missense & aHUS \\
\hline
\end{tabular}

Abbreviations: aHUS, atypical hemolytic uremic syndrome; HUS, hemolytic uremic syndrome, LP, likely pathogenic, P, pathogenic, TMA, thrombotic microangiopathy; VUS, variant of uncertain clinical significance.

${ }^{\mathrm{a}}$ Novel variant.

Table 3 Pathogenic and likely pathogenic variants

\begin{tabular}{|c|c|c|c|c|c|c|c|c|c|}
\hline Diagnosis & Gene & Variant (coding) & SIFT & PolyPhen & PROVEAN & $M A F^{\mathrm{a}}$ & GERP & $d b S N P$ & Human Splicing Finder \\
\hline HUS and TMA & $C D 46$ & c. $287-2 \mathrm{~A}>\mathrm{G}$ & N/A & N/A & N/A & $3.121 \mathrm{e}-05$ & 4.07 & No & Positive $^{\mathrm{b}}$ \\
\hline TMA & $C D 46$ & c. $1127+2 \mathrm{~T}>\mathrm{G}^{\mathrm{c}}$ & N/A & N/A & N/A & 0 & 2.96 & No & Positive $^{b}$ \\
\hline HUS & $C D 46$ & p.C35Y & 0 & 1 & -10.16 & 0 & 3.72 & No & N/A \\
\hline aHUS & $\mathrm{CFH}$ & c. $619+1 \mathrm{G}>\mathrm{A}^{\mathrm{c}}$ & N/A & N/A & N/A & 0 & 5.21 & No & Positive $^{b}$ \\
\hline aHUS & CFI & p.I370N & 0 & 0.998 & -6.09 & 0 & 5.71 & No & N/A \\
\hline HUS & C3 & p.R161W & 0.001 & 0.952 & -5.06 & 0 & 0.356 & No & N/A \\
\hline aHUS & C3 & p.R1042L & 0 & 0.996 & -6.16 & 0 & 5.76 & No & N/A \\
\hline aHUS & $D G K E$ & p.W332a & N/A & $\mathrm{N} / \mathrm{A}$ & N/A & $5.766 e-05$ & 5.59 & Rs138924661 & N/A \\
\hline
\end{tabular}

Abbreviations: aHUS, atypical hemolytic uremic syndrome; ExAC, Exome Aggregation Consortium; MAF, minor allele frequency; N/A, not applicable; TMA, thrombotic microangiopathy.

${ }^{a}$ Refers to the overall MAF in the ExAC database.

${ }^{\mathrm{b}}$ Alteration of wild-type acceptor site, most probably affecting splicing.

${ }^{\mathrm{C}}$ Novel variant.

previously. ${ }^{40,41}$ The patient with the previously reported p.I306V variant also harbored a homozygous deletion in CFHR3-CFHR1.

Six distinct variants were identified in C3, two likely pathogenic and four variants of uncertain clinical significance (Figure 2 and Table 4). Five were previously reported in the literature and/or the FH atypical hemolytic uremic syndrome database. $^{4,24,42}$ One patient with a likely pathogenic variant, p.R1042L, also carried the p.A43T variant of uncertain clinical significance in THBD.

$T H B D$ harbored variants in four patients (Figure 2). Three patients carried the p.A43T variant and one carried the p.P495S variant, both of uncertain clinical significance. Both variants have been previously reported and have low minor allele frequency $(<0.4 \%){ }^{43}$ As mentioned previously, one patient with a p.A43T variant also harbored a likely pathogenic variant in $C 3$. Another patient with the p.A43T variant carried a pathogenic variant in $D G K E$.

The only variant identified in DGKE, p.W322*, has been previously described in the literature as pathogenic (Figure 2 and Tables 2 and 3). ${ }^{44}$

\section{Diagnostic Yield}

Current ACMG/AMP guidelines indicate that variants classified as of uncertain clinical significance are not to be used in clinical decision making. ${ }^{25}$ 
Table 4 Variants of uncertain clinical significance

\begin{tabular}{|c|c|c|c|c|c|c|c|c|}
\hline Disease & Gene & Variant (coding) & SIFT & PolyPhen & PROVEAN & $M A F^{\mathrm{a}}$ & GERP & $d b S N P$ \\
\hline aHUS & $C D 46$ & p.P324L & 0.094 & 0.998 & -2.10 & 0.0006365 & -0.557 & Rs41317833 \\
\hline TMA & $\mathrm{CFH}$ & p.I372 $\mathrm{V}^{\mathrm{b}}$ & 0.025 & 0.981 & -0.72 & Not present & -7.48 & No \\
\hline aHUS & $\mathrm{CFH}$ & p.Q950H & 0.006 & 0.986 & -2.98 & 0.003583 & -4.92 & Rs149474608 \\
\hline HUS and TMA & CFH & p.G918E & 0.042 & 0.992 & -5.90 & Not present & 6.04 & No \\
\hline HUS & CFI & p.I306V & 0.458 & 0.012 & 0.08 & 0.0004798 & -2.55 & Rs113273712 \\
\hline aHUS & CFI & p.D403N & 1 & 0.003 & 1.45 & $2.48 \mathrm{e}-05$ & 0.0847 & Rs139881195 \\
\hline HUS & C3 & p.T647M & 0.018 & 0.321 & -1.13 & Not present & 2.63 & No \\
\hline aHUS & C3 & p.Q185H & 0.065 & 0.033 & -1.36 & Not present & -1.4 & No \\
\hline aHUS & C3 & p.T1383N & 0.036 & 0.63 & -1.62 & $9.06 e-05$ & 1.59 & Rs139100972 \\
\hline aHUS & C3 & p.E1636G & 0.002 & 0.967 & -5.55 & Not present & 3.95 & No \\
\hline HUS & $T H B D$ & p.P495S & 0.146 & 0.273 & -2.74 & 0.000575 & -0.884 & Rs1800578 \\
\hline aHUS $\times 3$ patients & THBD & p.A43T & 0.533 & 0.002 & -0.39 & 0.00343 & 1.61 & Rs1800576 \\
\hline
\end{tabular}

Abbreviations: aHUS, atypical hemolytic uremic syndrome; ExAC, Exome Aggregation Consortium; HUS, hemolytic uremic syndrome; MAF, minor allele frequency; TMA, thrombotic microangiopathy.

${ }^{\mathrm{a}}$ Refers to the overall MAF in the ExAC database.

b Novel variant.

${ }^{\mathrm{C}}$ Identified in association with a pathogenic variant in $C D 46$.

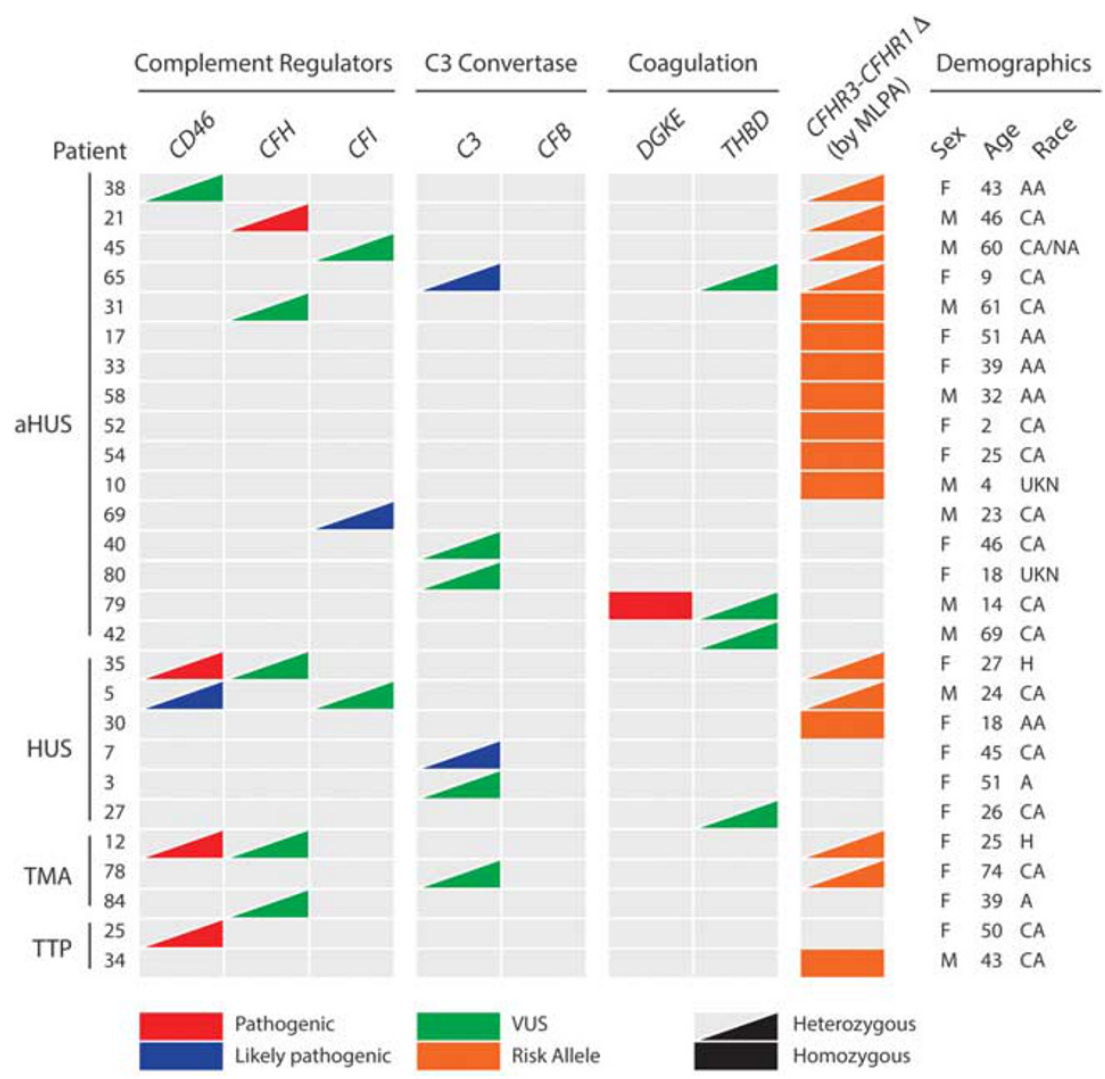

Figure 2 Summary of thrombotic microangiopathy genetic testing results. Patients are grouped according to clinical diagnosis. Genes are grouped according to function. Age represents the age at the time of testing. A, Asian, AA, African American; aHUS, atypical hemolytic uremic syndrome, CA, Caucasian; H, Hispanic; HUS, hemolytic uremic syndrome; NA, Native American; TMA, thrombotic microangiopathy; TTP, thrombotic thrombocytopenic purpura; Unk, unknown; VUS, variant of uncertain clinical significance.

Therefore, these are not considered a positive genetic diagnosis in this study for purposes of measuring clinical utility or diagnostic yield. In patients with a clinical diagnosis of thrombotic microangiopathy, atypical hemolytic uremic syndrome, hemolytic uremic syndrome, or thrombotic thrombocytopenic purpura $(n=73), 9$ had pathogenic or likely pathogenic variants, and 9 had homozygous deletions of CFHR3-CFHR1, representing a diagnostic yield of $25 \%$ (18/73). Isolated variants of uncertain clinical significance were identified in 10 (14\%) patients. 


\section{Discussion}

We report our 1-year experience using exome-based next-generation sequencing in routine diagnostic evaluation of patients referred for thrombotic microangiopathy genetic testing. Our results showed pathogenic or likely pathogenic variants in $12 \%$ (9/73) and variants of uncertain clinical significance in $21 \%(15 / 73)$ of all patients tested. This is comparable to the rate of pathogenic and likely pathogenic variants identified in a previous large series of patients sequenced for thrombotic microangiopathy. ${ }^{3}$ In that particular study, 193 patients were sequenced, and 23 pathogenic or likely pathogenic variants were identified for a diagnostic yield of $12 \% .^{3}$ In our current study, when homozygous deletions of CFHR3-CFHR1 are included, the overall diagnostic yield increases to $21 \%$. Similarly, in the large series by $\mathrm{Bu}$ et al., ${ }^{3}$ including the rate of homozygous deletions of CFHR3-CFHR1 increases their diagnostic yield to $21 \%$. The current study shows that exome-based, targeted next-generation sequencing sequencing in a CAP/CLIA-certified laboratory is an efficient and reliable approach for the identification of diagnostic variants in patients with thrombotic microangiopathy. The positive diagnostic rate is comparable to a similar series that used non-exome-based next-generation sequencing sequencing techniques. ${ }^{3}$

Genetic sequencing has the potential to uncover single-nucleotide variants of clinical significance that have not been previously described in the literature or reported in existing databases. A significant finding of our study is the identification of five such novel variants. Three of these are pathogenic or likely pathogenic based on standardized interpretation guidelines. ${ }^{25}$ In addition to revealing new pathogenic variants, comprehensive gene sequencing reveals novel variants, and currently undefinable variants, classified as variants of uncertain clinical significance.

In all patients referred for thrombotic microangiopathy genetic testing, variants of uncertain clinical significance represent the largest category of singlenucleotide variants identified at $22=1 \%(15 / 73)$. However, this is significantly lower than the rate of variants of uncertain clinical significance previously reported by $\mathrm{Bu}$ et $a .^{3}{ }^{3}$ of $35 \%(68 / 193)(P=0.026)$. A portion of this discrepancy may reflect the absence of CFHR5 and PLG in our reportable gene set. However, this discordance may be further attributed to differences in criteria applied to classify variants between the two studies. In the current study, comparison of variant classifications showed significantly higher GERP++ scores and lower minor allele frequency in pathogenic/likely pathogenic variants compared with variants of uncertain clinical significance. Similarly, in silico prediction tools showed significant differences between these two groups.
In the current series, patients referred for thrombotic microangiopathy genetic sequencing most commonly harbor single-nucleotide variants in $C F H$, but variants identified in $C D 46$ are most likely to be classified as pathogenic owing to the higher incidence of splice-site variants identified in the gene. Homozygous CFHR3-CFHR1 deletions are identified in $12 \%$ of patients, indicating an increased risk for the development of anticomplement factor $\mathrm{H}$ antibodies. ${ }^{45}$

Resolving variants of uncertain clinical significance is challenging for clinicians owing to less than definitive information on the variant and its application to patient management. In such settings, discussions with a clinical team composed of molecular pathologists, geneticists, genetic counselors, and nephrologists may be useful. Current recommendations indicate that variants of uncertain clinical significance should not be used in clinical decision making. ${ }^{25}$ Therefore, to further define associations between gene variants and pathogenesis, genetic findings need to be correlated with functional studies, clinical outcome, pathology findings, familial cosegregation, and treatment response. ${ }^{25}$ Renal genetic analysis is challenging due to incomplete penetrance, variable expressivity, and complex modes of inheritance. It is anticipated that as more laboratories describe genetic variants, further studies will be performed to illuminate their significance.

In addition to follow-up analyses, our data suggest a need for expanded variant curation resources in the setting of renal disease including a centralized database to deposit annotated disease-associated variants identified as part of routine clinical care. Presently, the ClinVar database serves as a public archive documenting the report of relationships between human phenotype and genetic variation. However, the paucity of renal disease-associated variation makes this resource limited in function. Expansion of the ClinVar database and other renal genetic knowledgebases would be invaluable to assist laboratories in assigning significance to individual variants with little published information. Such a database needs accurate clinical information in addition to variant information and could serve as a source for other investigators to use in attempts to further characterize unique genetic findings.

New disease gene associations continue to emerge. Chong et al. ${ }^{46}$ report 915 new disease genes over the past 4 years. ${ }^{46}$ It is therefore important to design a test readily adaptable to new discoveries without incurring significant cost and time. Using a unique panel-based next-generation sequencing test requires revalidation for each new gene target added. In contrast, building a test on an exome backbone allows for simpler and more rapid validation of new genes, making this approach nimble and costeffective. Using this approach, clinically relevant genes are reported in the medical record, while the remaining exome sequence is captured and bioinformatically masked. Thus, these data can be 
leveraged as new biologic gene targets emerge. Under appropriate consent/human research protections, these data also provide a rich resource of research material to investigate additional genes associated with thrombotic microangiopathy and potential genetic modifiers contributing to states of inappropriate complement activation.

The study is limited by the small sample size, an inherent problem when studying rare diseases such as atypical hemolytic uremic syndrome. Since many of the cases are referred from outside institutions, complete clinical data are not available for majority of patients. Reliance on the referring clinician's diagnostic impression may artificially lower the diagnostic yield. Nonetheless, this reflects the results clinicians can expect in actual clinical practice.

This study describes the use of clinical exomebased next-generation sequencing testing with targeted bioinformatics analysis and reporting for routine care of patients referred for thrombotic microangiopathy genetic testing. Our results highlight the inherent difficulty in applying the current ACMG/AMP guidelines in interpreting singlenucleotide variants due to the paucity of available data in the renal disease setting. The results show a lower diagnostic yield than previously reported and a high number of variants of uncertain clinical significance. The decrease in diagnostic yield appears to be due to the absence of variants of uncertain clinical significance for inclusion as a positive diagnostic finding. The current study demonstrates that exome-based next-generation sequencing testing in patients with thrombotic microangiopathies can be applied in a clinical setting and provide meaningful results to assist in clinical care of patients.

\section{Acknowledgments}

We thank Kidney Translational Research Core (KTRC), partly supported by DK079333, for providing samples for validation testing. This work was previously presented at the United States and Canadian Academy of Pathology Annual Meeting in Seattle, WA in March of 2016. All authors reviewed the data and participated in preparation of the manuscript and approved the final version.

\section{Disclosure/conflict of interest}

JDP is a cofounder of PierianDx JWH is a paid consultant for PierianDx, and JPG is a member of the Alexion Genetic Testing Advisory Board.

\section{References}

1 Nester CM, Barbour T, de Cordoba SR, et al. Atypical aHUS: state of the art. Mol Immunol 2015;67:31-42.
2 George JN, Nester CM. Syndromes of thrombotic microangiopathy. N Engl J Med 2014;371:654-666.

$3 \mathrm{Bu} \mathrm{F}$, Borsa NG, Jones $\mathrm{MB}$, et al. High-throughput genetic testing for thrombotic microangiopathies and C3 glomerulopathies. J Am Soc Nephrol 2016;27: 1245-1253.

4 Noris M, Caprioli J, Bresin E, et al. Relative role of genetic complement abnormalities in sporadic and familial aHUS and their impact on clinical phenotype. Clin J Am Soc Nephrol 2010;5:1844-1859.

5 Noris M, Remuzzi G. Atypical hemolytic-uremic syndrome. N Engl J Med 2009;361:1676-1687.

6 Greenbaum LA. Atypical hemolytic uremic syndrome. Adv Pediatr 2014;61:335-356.

7 Fremeaux-Bacchi V, Fakhouri F, Garnier A, et al. Genetics and outcome of atypical hemolytic uremic syndrome: a nationwide French series comparing children and adults. Clin J Am Soc Nephrol 2013;8: $554-562$.

$8 \mathrm{Bu} \mathrm{F}$, Borsa N, Gianluigi A et al. Familial atypical hemolytic uremic syndrome: a review of its genetic and clinical aspects. Clin Dev Immunol 2012;2012:370426.

9 Kavanagh D, Goodship TH, Richards A. Atypical hemolytic uremic syndrome. Semin Nephrol 2013;33: 508-530.

10 Stokman MF, Renkema KY, Giles RH, et al. The expanding phenotypic spectra of kidney diseases: insights from genetic studies. Nat Rev Nephrol 2016;12:472-483.

11 Vivante A, Hildebrandt F. Exploring the genetic basis of early-onset chronic kidney disease. Nat Rev Nephrol 2016;12:133-146.

12 Prakash S, Gharavi AG. Diagnosing kidney disease in the genetic era. Curr Opin Nephrol Hypertens 2015;24: 380-387.

13 Fakhouri F, Fila M, Provot F, et al. Pathogenic variants in complement genes and risk of atypical hemolytic uremic syndrome relapse after eculizumab discontinuation. Clin J Am Soc Nephrol 2016;12:50-59.

$14 \mathrm{Bu} \mathrm{F}$, Maga T, Meyer NC, et al. Comprehensive genetic analysis of complement and coagulation genes in atypical hemolytic uremic syndrome. J Am Soc Nephrol 2014;25:55-64.

15 Taylor CM, Machin S, Wigmore SJ, et al. Clinical practice guidelines for the management of atypical haemolytic uraemic syndrome in the United Kingdom. Br J Haematol 2010;148:37-47.

16 Campistol JM, Arias M, Ariceta G, et al. An update for atypical haemolytic uraemic syndrome: diagnosis and treatment. A consensus document. Nefrologia 2015;35: 421-447.

17 Loirat C, Fakhouri F, Ariceta G, et al. An international consensus approach to the management of atypical hemolytic uremic syndrome in children. Pediatr Nephrol 2016;31:15-39.

18 Wetterstrand K. Sequencing costs: data from the NHGRI genome sequencing program (GSP). Available at: https://www.genome.gov/sequencingcostsdata/ (last accessed 9 August 2016).

19 Braun DA, Schueler M, Halbritter J, et al. Whole exome sequencing identifies causative mutations in the majority of consanguineous or familial cases with childhoodonset increased renal echogenicity. Kidney Int 2016;89: 468-475.

20 Mori T, Hosomichi K, Chiga M, et al. Comprehensive genetic testing approach for major inherited kidney diseases, using next-generation sequencing with a custom panel. Clin Exp Nephrol 2016;21:63-75. 
21 Moriniere V, Dahan K, Hilbert P, et al. Improving mutation screening in familial hematuric nephropathies through next generation sequencing. J Am Soc Nephrol 2014;25:2740-2751.

22 Chatterjee R, Ramos E, Hoffman M, et al. Traditional and targeted exome sequencing reveals common, rare and novel functional deleterious variants in RET-signaling complex in a cohort of living US patients with urinary tract malformations. Hum Genet 2012;131: 1725-1738.

23 Liapis H, Jain S. The interface of genetics with pathology in alport nephritis. J Am Soc Nephrol 2013;24:1925-1927.

24 Maga TK, Nishimura CJ, Weaver AE et al. Mutations in alternative pathway complement proteins in American patients with atypical hemolytic uremic syndrome. Hum Mutat 2010;31:E1445-E1460.

25 Richards S, Aziz N, Bale S, et al. Standards and guidelines for the interpretation of sequence variants: a joint consensus recommendation of the American College of Medical Genetics and Genomics and the Association for Molecular Pathology. Genet Med 2015;17:405-424.

26 Schouten JP, McElgunn CJ, Waaijer R, et al. Relative quantification of 40 nucleic acid sequences by multiplex ligation-dependent probe amplification. Nucleic Acids Res 2002;30:e57.

27 Moore I, Strain L, Pappworth I, et al. Association of factor $\mathrm{H}$ autoantibodies with deletions of CFHR1, CFHR3, CFHR4, and with mutations in $\mathrm{CFH}$, CFI, CD46, and $\mathrm{C} 3$ in patients with atypical hemolytic uremic syndrome. Blood 2010;115: 379-387.

28 Aziz N, Zhao Q, Bry L, et al. College of American Pathologists' laboratory standards for next-generation sequencing clinical tests. Arch Pathol Lab Med 2015;139:481-493.

29 Sharma MK, Phillips J, Agarwal S, et al. Clinical genomicist workstation. AMIA Jt Summits Transl Sci Proc 2013;2013:156-157.

30 Lek M, Karczewski KJ, Minikel EV, et al. Analysis of protein-coding genetic variation in 60,706 humans. Nature 2016;536:285-291.

$31 \mathrm{Li} \mathrm{H}$. A statistical framework for SNP calling, mutation discovery, association mapping and population genetical parameter estimation from sequencing data. Bioinformatics 2011;27:2987-2993.

32 Li H, Handsaker B, Wysoker A, et al. The Sequence Alignment/Map format and SAMtools. Bioinformatics 2009;25:2078-2079.

33 Davydov EV, Goode DL, Sirota M, et al. Identifying a high fraction of the human genome to be under selective constraint using GERP++. PLoS Comput Biol 2010;6:e1001025.

34 Choi Y, Sims GE, Murphy S, Miller JR, Chan AP. Predicting the functional effect of amino acid substitutions and indels. PLoS ONE 2012;7:e46688.

35 Caprioli J, Castelletti F, Bucchioni S, et al. Complement factor $\mathrm{H}$ mutations and gene polymorphisms in haemolytic uraemic syndrome: the C-257T, the A2089G and the G2881T polymorphisms are strongly associated with the disease. Hum Mol Genet 2003;12:3385-3395.

36 Mohlin FC, Nilsson SC, Levart TK, et al. Functional characterization of two novel non-synonymous alterations in CD46 and a Q950H change in factor $\mathrm{H}$ found in atypical hemolytic uremic syndrome patients. Mol Immunol 2015;65:367-376.

37 Neumann HP, Salzmann M, Bohnert-Iwan B, et al. Haemolytic uraemic syndrome and mutations of the factor $\mathrm{H}$ gene: a registry-based study of German speaking countries. J Med Genet 2003;40:676-681.

38 Perez-Caballero D, Gonzalez-Rubio C, Gallardo ME, et al. Clustering of missense mutations in the C-terminal region of factor $\mathrm{H}$ in atypical hemolytic uremic syndrome. Am J Hum Genet 2001;68:478-484.

39 Sartz L, Olin AI, Kristoffersson AC, et al. A novel C3 mutation causing increased formation of the C3 convertase in familial atypical hemolytic uremic syndrome. J Immunol 2012;188:2030-2037.

40 Le Quintrec M, Lionet A, Kamar N, et al. Complement mutation-associated de novo thrombotic microangiopathy following kidney transplantation. Am J Transplant 2008;8:1694-1701.

41 Bienaime F, Dragon-Durey MA, Regnier CH, et al. Mutations in components of complement influence the outcome of factor I-associated atypical hemolytic uremic syndrome. Kidney Int 2010;77:339-349.

42 Schramm EC, Roumenina LT, Rybkine $\mathrm{T}$, et al. Mapping interactions between complement C3 and regulators using mutations in atypical hemolytic uremic syndrome. Blood 2015;125:2359-2369.

43 Delvaeye M, Noris M, De Vriese A, et al. Thrombomodulin mutations in atypical hemolytic-uremic syndrome. N Engl J Med 2009;361:345-357.

44 Lemaire M, Fremeaux-Bacchi V, Schaefer F, et al. Recessive mutations in DGKE cause atypical hemolyticuremic syndrome. Nat Genet 2013;45:531-536.

45 Dragon-Durey MA, Loirat C, Cloarec S, et al. Anti-factor $\mathrm{H}$ autoantibodies associated with atypical hemolytic uremic syndrome. J Am Soc Nephrol 2005;16:555-563.

46 Chong JX, Buckingham KJ, Jhangiani SN, et al. The genetic basis of mendelian phenotypes: discoveries, challenges, and opportunities. Am J Hum Genet 2015;97:199-215. 